\title{
STATUS OF THE FERMILAB MAIN INJECTOR
}

\author{
K.Koba for the Main Injector group \\ FNAL, Batavia, IL 60510, USA
}

\begin{abstract}
The Fermilab Main Injector is a rapid cycling proton synchrotron. It is designed to accelerate protons and antiprotons to $150 \mathrm{GeV}$. The initial commissioning phase was in the summer of 1999. Since then, Main Injector has been supporting the high energy physics program at Fermilab. Beam studies for continued improvements in machine performance are in progress, in order to support a luminosity of $8 * 10^{31} \mathrm{~cm}^{-2} \mathrm{sec}^{-1}$ during Run IIa. The status of the Main Injector and beam studies results are presented.
\end{abstract}

\section{INTRODUCTION}

Proton and antiproton beams are accelerated in Main Injector to $150 \mathrm{GeV}$ and extracted to the Tevatron. Current high luminosity collider operation, which is called Run IIa, has already started in the summer in 2000 and has a luminosity goal of $8 * 10^{31} \mathrm{~cm}^{-2} \mathrm{sec}^{-1}$. The Tevatron collides 36 proton bunches with 36 antiproton bunches. These numbers signify that the required intensity for the Main Injector is $2.7^{*} 10^{11}$ particles per bunch (ppb) for protons and $3.0^{*} 10^{10} \mathrm{ppb}$ for antiprotons. The required longitudinal emittance is less than $3 \mathrm{eV}$-sec for both of types of bunch.

Run IIb will follow Run IIa and has a luminosity goal $5.2 * 10^{32} \mathrm{~cm}^{-2} \mathrm{sec}^{-1}$. For Run IIb, $1.1 * 10^{13}$ total antiprotons will be required.

Main Injector also accelerates protons to $120 \mathrm{GeV}$ for pbar production. The required intensity for pbar production is $5.0^{*} 10^{12}$ in 84 bunches.

Thus, an increasingly intense beam is needed over the next 5 years. Currently, several beam studies are underway to get a beam high line density in Main Injector. Typical Main Injector machine parameter is listed on Table 1.

Table 1: Main parameter of the Main Injector

\begin{tabular}{|l|l|}
\hline Injection energy & $8.0 \mathrm{GeV}$ \\
\hline Extraction energy & $150 \mathrm{GeV}, 120 \mathrm{GeV}$ \\
\hline Harmonic number & 588 \\
\hline Mean Radius & $528.3 \mathrm{~m}$ \\
\hline Transition gamma & 21.6 \\
\hline
\end{tabular}

\section{OPERATING CONDITIONS}

There are 3 typical cycles for the collider operation[1].

\section{1 proton acceleration}

Seven proton bunches which an energy of $8.0 \mathrm{GeV}$ are injected from the Booster. These bunches are accelerated to $150 \mathrm{GeV}$, concentrated into one bunch, and extracted to the Tevatron. This cycle is repeated 36 times in order to inject 36 bunches in the Tevatron. The typical intensity of an extracted bunch is $2.5^{*} 10^{11} \mathrm{ppb}$, with an emittance of $3 \mathrm{eV}$-sec. This operation is shown in Fig. 1.

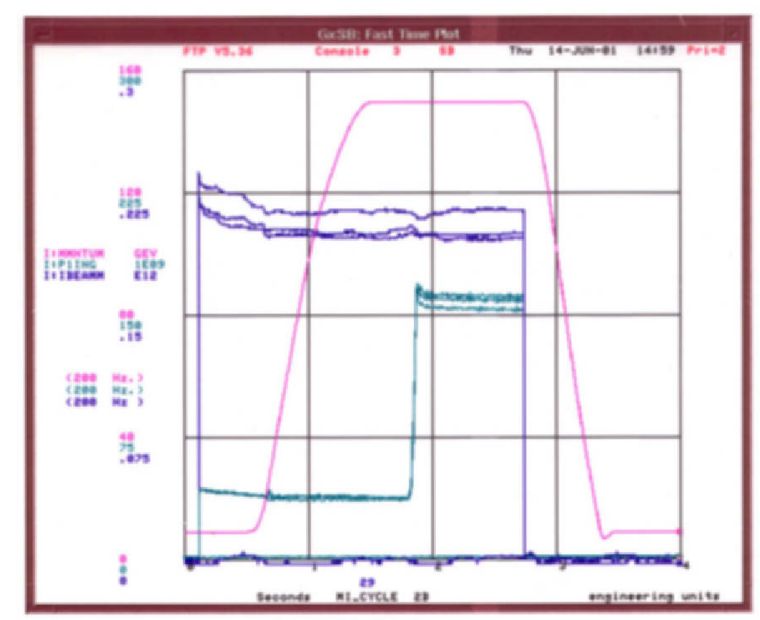

Figure 1: Operation of the proton acceleration cycle. (1) Momentum, (2) Total intensity, (3) Intensity of the one RF bucket

\section{2 antiproton production}

84 proton bunches which have an energy of $8.0 \mathrm{GeV}$ are injected from the Booster. These bunches are accelerated to $120 \mathrm{GeV}$, extracted to the Antiproton line, and hit the target to produce antiprotons. This operation is shown in Fig. 2.

The Main Injector is working well with a rapid cycling rate of $1.5 \mathrm{sec}$, and an intensity of $4.2 * 10^{12}$ particles per pulse. This performance is better than achieved previously in the Main Ring synchrotron, which had a peak intensity of $3.2 * 10^{12} \mathrm{ppp}$ and minimum cycling rate of $2.4 \mathrm{sec}$.

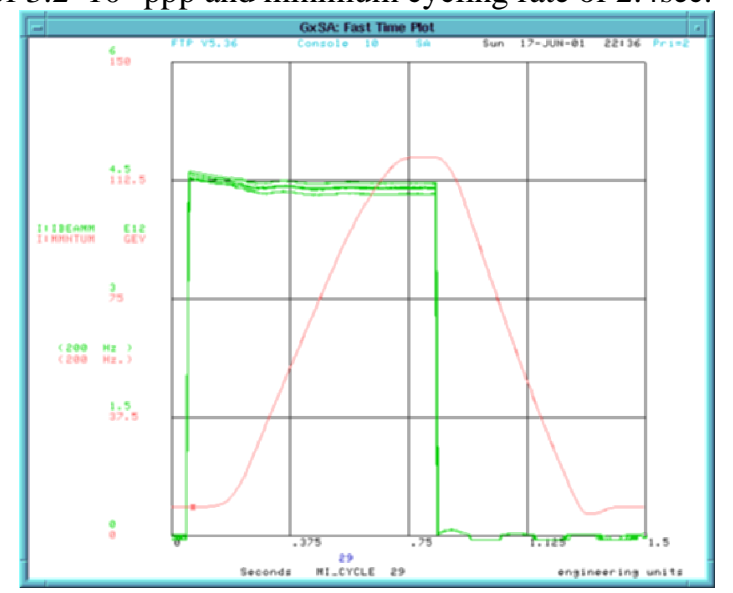

Figure 2: Operation of the antiproton production cycle. (1) Momentum, (2) Total intensity 


\section{3 antiproton acceleration}

Four antiproton batches with each batch consisting of 7 bunches are injected from the accumulator ring with an energy of $8.0 \mathrm{GeV}$. These batches are accelerated to $150 \mathrm{GeV}$ and each batch is concentrated into one bunch. Finally, 4 bunches are extracted to the Tevatron. This cycle is repeated 9 times to inject 36 bunches in Tevatron. The typical intensity of an extracted bunch is $2.5^{*} 10^{10} \mathrm{ppb}$, and the emittance is $3 \mathrm{eV}$-sec. This operation is shown in Fig. 3.

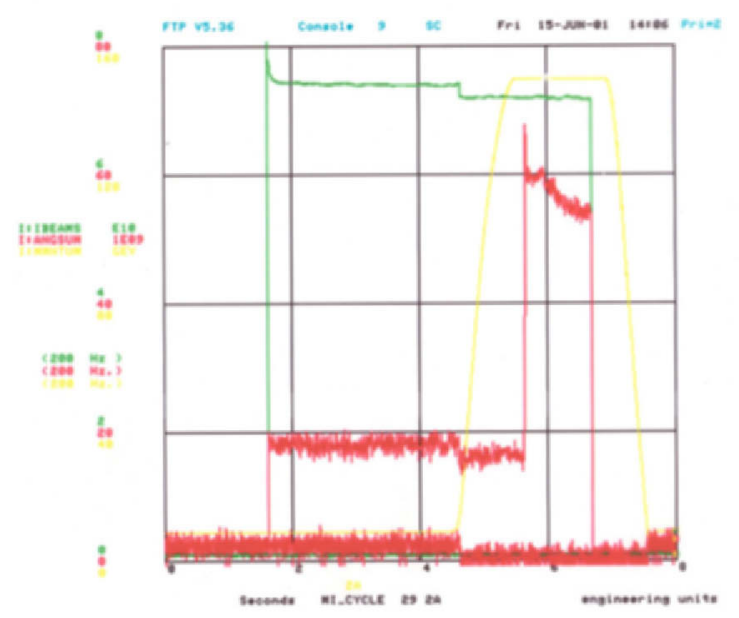

Figure 3: Operation of the proton acceleration cycle. (1) Momentum, (2) Total intensity, (3) Intensity of the one RF bucket

\section{BEAM STUDIES FOR THE LUMINOSITY UPGRADE}

Several beam studies are underway to help us achieve the Run II intensity and longitudinal emittance goals.

\subsection{Coalescing}

Coalescing[2][3] is a process in which several bunches are concentrated into one bunch by using lower frequency RF. In the standard operation, we take the following four steps:

Step I: Five to Seven bunches are circulating while captured by an RF system with a frequency of $53 \mathrm{MHz}$ and a voltage of 1.1MV. The bunch shape matches the 1.1MV RF bucket.

Step II: The voltage is decreased to $35 \mathrm{kV}$ suddenly, so the bunches start rotating in phase space. After a quarter of a synchrotron period, the momentum spread is at a minimum.

Step III: The $53 \mathrm{MHz}$ RF voltage is changed to $0 \mathrm{~V}$. At the same time, a $2.5 \mathrm{MHz}$ RF system with a voltage of $75 \mathrm{kV}$ is brought on. The bunches start rotating in the $2.5 \mathrm{MHz}$ RF bucket. After a quarter of a synchrotron period, the bunch length becomes minimum.

Step IV: The $2.5 \mathrm{MHz}$ RF voltage is changed to $0 \mathrm{~V}$. At the same time, the $53 \mathrm{MHz}$ RF voltage is increased to $1.1 \mathrm{MV}$ and captures the coalesced bunch.
Figure 4 shows the transformation of the bunch shape during coalescing. The efficiencies of proton and antiproton coalescing are $70 \%$ and $80 \%$, respectively. We are trying to get greater efficiency by using a higher harmonic cavity, which operates at $106 \mathrm{MHz}$ for Step II and $5 \mathrm{MHz}$ for Step III[4][5][6].

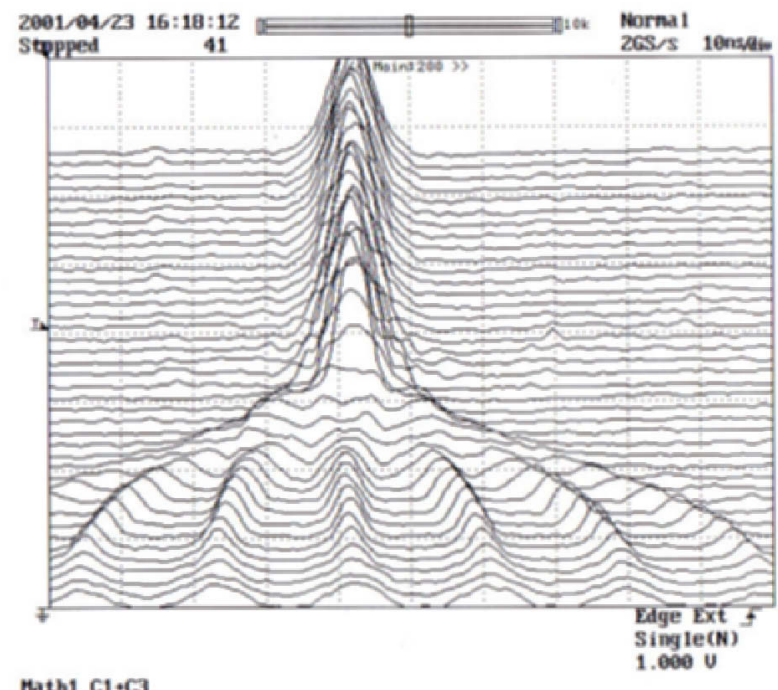

Figure 4: Measured bunch transformation during the antiproton coalescing.

\subsection{Slip stacking}

For Run IIb operation, we are trying to increase the number of protons on the antiproton target during the antiproton production cycle. Slip stacking[7] is a process whereby two circulating batches are concentrated into one batch.

Step I: The first batch is injected from Booster and slightly accelerated to change the circulating orbit. The frequency of the first batch after acceleration is $f_{0}+\Delta f$.

Step II: A second batch is injected and slightly decelerated. The second decelerated batch has a frequency of $f_{0}-\Delta f$.

Step III: The first batch is then decelerated and $2^{\text {nd }}$ batch is accelerated and both are captured by one RF bucket with the frequency of $f_{0}$.

We have already started beam studies, having done momentum aperture scans and the cogged the frequency as explained in Step I. Work will continue through 2001.

\subsection{Beam loading compensation}

For both RF manipulations, coalescing and slip stacking, beam loading compensation is a key issue. An RF feedback system has already been implemented with a gain of $20 \mathrm{~dB}$. The gap voltage phase shift signals with and without feedback are shown in Fig. 5 and Fig. 6, respectively. Using this decay rate, Q-value of 480 was calculated. This Q-value was consistent with the offline measurement[8]. 
We are working on the development of a feed foreword system and also on increasing the gain of the RF feedback[9][10].

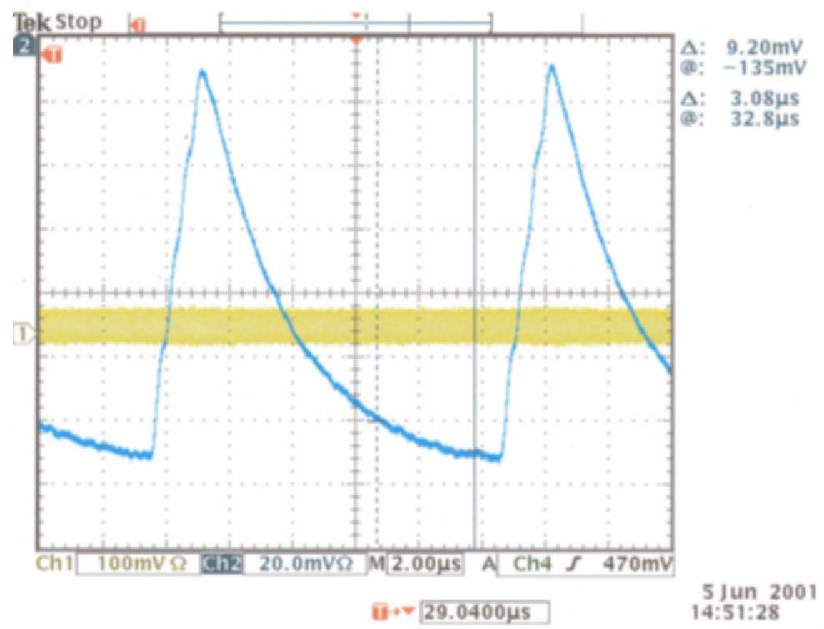

Figure 5: The gap voltage phase shift with feedback.

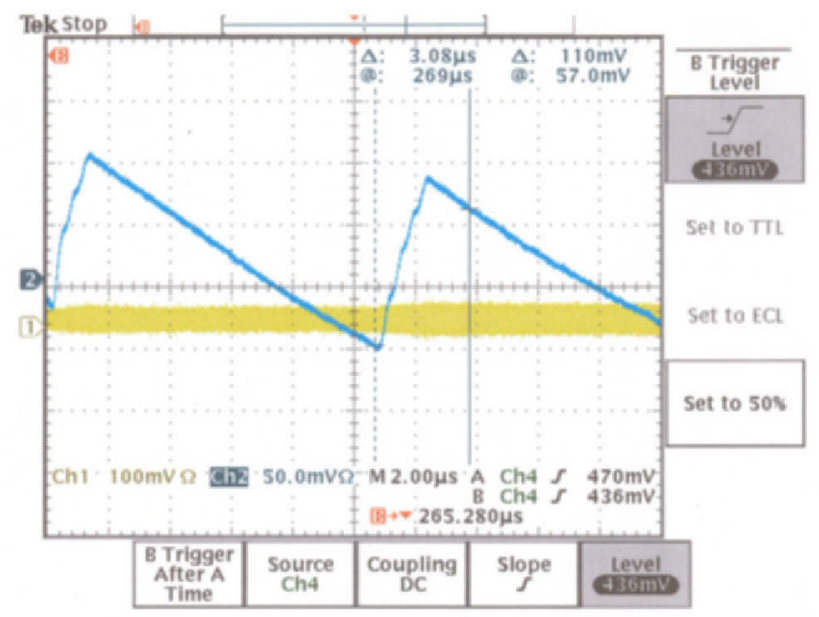

Figure 6: The gap voltage phase shift without feedback.

\section{CONCLUSION}

Main Injector performance is critical for RUN II collider operation. Main Injector is already providing beam for Run II collider operation. Improvement in coalescing and slip stacking are essential to achieving the Run II luminosity goals. Beam loading compensation is needed for all the Main Injector RF manipulations.

\section{REFERENCES}

[1] Main Injector Rookie Book.

[2] J.E. Griffin et al, "Preparation and study of bunches containing $10^{11}$ protons in the Fermilab Main Ring.", IEEE, 1981.

[3] I. Kourbanis et al, "Performance and comparison of different coalescing scheme used in Fermilab Main Ring", Proceedings of 1993 PAC, 1993.

[4] J.Dey et al, "A New RF system for bunch coalescing in the Fermilab Main Ring.", Proceedings of PAC, 1996.

[5] J.Dey et al, "Improvements in bunch coalescing in the Fermilab Main Ring.", Proceedings of PAC, 1996.

[6] J.Dey et al, " $106 \mathrm{MHz}$ cavity for improving coalescing efficiency in the Fermilab Main Ring.", Proceedings of PAC, 1996.

[7] S. Shukla et al, "Slip Stacking in the Fermilab Main Injector.", Snowmass'96, June 1996.

[8] K. Koba et al, "Beam loading compensation requirement for multibatch coalescing in Fermilab", these proceedings.

[9] J.Dey and J.Steimel, "Improving the linearity of ferrite loaded cavities using feedback", these proceedings

[10] J.Dey, J. Steimel and J. Reid, "Narrowband Beam Loading Compensation in the Fermilab Main Injector Accelerating Cavities", these proceeding 